\title{
Adenine Nucleotide Pool and Energy Charge During Growth of a Tyrothricin-producing Strain of Bacillus brevis
}

\author{
By G. H. FYNN AND J. A. DAVISON \\ Department of Biochemistry, University of Manchester Institute \\ of Science and Technology, Manchester M60 I $Q D$ \\ (Received II July 1975; revised I4 November 1975)
}

\begin{abstract}
SUMMARY
The adenine nucleotide levels and derived energy charge value of a tyrothricinproducing strain of Bacillus brevis under aerobic conditions were in good agreement with published values for other bacteria. When growing cultures of $B$. brevis underwent a transition from aerobic to anaerobic conditions, cyclic variations in the level of adenine nucleotides were observed and the energy charge value oscillated between 0.87 and 0.70 . The significance of these changes is considered in relation to antibiotic production as a possible regulatory mechanism in energy metabolism. It is concluded that tyrothricin is not directly involved in the observed changes in energy charge value.
\end{abstract}

\section{INTRODUCTION}

Silaeva et al. (1965) reported that very low levels of ATP and ADP and a relatively high level of AMP were present in the nucleotide pool of gramicidin S-producing $(+)$ strains of Bacillus brevis GB. By comparison, higher concentrations of ATP were found in the non-gramicidin S-producing (-) strain, although the amount was still low relative to the AMP level. An efflux of nucleotide into the growth medium was also observed during growth of the antibiotic-producing $(+)$ strain but not during growth of the $(-)$ strain. The presence of $\beta$-phenyl- $\beta$-alanine in the culture medium inhibited antibiotic synthesis and under these conditions the $(+)$ strain exhibited a nucleotide pool composition and nucleotide retention pattern characteristic of the $(-)$ strain. Conversely, the addition of gramicidin $S$ to the growth medium of the $(-)$ strain altered the nucleotide pool characteristics to resemble that of the $(+)$ strain. On the basis of the observed nucleotide levels it was concluded by Silaeva and co-workers that oxidative phosphorylation was not significant in energy production in B. brevis.

Atkinson (1968) first introduced the concept of energy charge (E.C.) value which describes the relative levels of ATP, ADP and AMP in the nucleotide pool. In terms of molar concentrations, the energy charge is defined as

$$
\text { E.C. }=\left[(\mathrm{ATP})+\frac{1}{2}(\mathrm{ADP})\right] /[(\mathrm{ATP})+(\mathrm{ADP})+(\mathrm{AMP})]
$$

The E.C. parameter has been investigated in a number of micro-organisms and values for intact growing cells appear to be strongly poised near 0.8 (Chapman, Fall \& Atkinson, 1971 ).

The previous findings reported for the GB strain of $B$. brevis suggest that the E.C. value for this organism is abnormally low. However, previous work in this laboratory (Fynn \& Seddon, 1971; Seddon \& Fynn, 1970, 1971; Fynn, Thomas \& Seddon, 1972) has shown that with $B$. brevis ATCC10068 (which produces tyrothricin rather than gramicidin $S$ ) there 
is an active terminal electron transport system as well as NAD-dependent dehydrogenase enzymes. Dinitrophenol and tyrothricin, which are uncouplers of oxidative phosphorylation, inhibited growth of the organism when added to the culture medium. Seddon \& Fynn (I973) proposed that the endogenous tyrothricin normally produced during growth is inactivated by compartmentalization or by binding with phospholipid within the cell. An investigation was therefore undertaken of the adenine nucleotide pool and E.C. values in Bacillus brevis ATCCI0068, both in the presence and absence of antibiotic production.

\section{METHODS}

Organism, growth and maintenance. Bacillus brevis ATCC10068 was grown on asparagineglycerol and peptone-yeast extract media under reciprocal shake conditions and growth was measured as previously described (Seddon \& Fynn, I97 I, 1973).

Materials. The enzymes and specialized chemicals used in this work were obtained from Sigma.

Extraction and assay of adenine nucleotides. Samples of the growing culture were denatured with $\mathrm{HClO}_{4}$, centrifuged and neutralized as previously described (Davison \& Fynn, 1974). The ATP content of the extracts was assayed using the luciferin-luciferase system. ADP and AMP present in the extract were first converted to ATP using the method of Chapman et al. (1971) and then assayed using the luciferin-luciferase system (Davison \& Fynn, 1974).

For the assay of ATP the assay mixture typically contained: neutralized extract, $0.2 \mathrm{ml}$; $\mathrm{MgCl}_{2}, 3.0 \mu \mathrm{mol}$; potassium phosphate buffer $\mathrm{pH} 7 \cdot 4,7.5 \mu \mathrm{mol}$; EDTA, I $\mu \mathrm{mol}$; and water to $\mathrm{I} \cdot 0 \mathrm{ml}$. Where ADP was to be measured, phosphoenolpyruvate $(5 \mu \mathrm{mol})$ and pyruvate kinase $(20 \mu \mathrm{g})$, were included in the assay mixture. For AMP assay the reaction mixture was as described for ATP plus ADP with the addition of adenylate kinase $(25 \mu \mathrm{g})$. All estimations were performed in duplicate, with appropriate blanks where water replaced the extract. The complete reaction mixtures were incubated at $37^{\circ} \mathrm{C}$ for $30 \mathrm{~min}$ then cooled in ice before measurement of the ATP content. ADP and AMP contents were calculated from the relevant ATP values by difference and E.C. values calculated from this data. Internal and external ATP standards were carried through the entire assay procedure and recovery values used to correct the experimental results.

Assay of extracellular alkaline phosphatase. A sample was withdrawn from the culture and the bacteria centrifuged at $10500 \mathrm{~g}$ for $2 \mathrm{~min}$. A portion of the supernatant was then incubated in the following mixture: Tris- $\mathrm{HCl}$ buffer $\mathrm{pH} 8.2,10 \mu \mathrm{mol} ; \mathrm{MgCl}_{2}, 6 \mu \mathrm{mol}$; supernatant, $0.2 \mathrm{ml}$; and water to $0.9 \mathrm{ml}$. Incubation at $37^{\circ} \mathrm{C}$ for $10 \mathrm{~min}$ served to reduce the concentration of phosphate to a low level. A sample ( $0.1 \mathrm{ml}$ ) of ATP (typically $50 \mathrm{nmol}$ ) was then introduced. At appropriate times, $0.1 \mathrm{ml}$ samples were withdrawn and the residual ATP immediately determined using the luciferin-luciferase system. A blank was included in each experiment in which the supernatant sample was replaced by $0.2 \mathrm{ml}$ of water, and this showed a negligible fall in ATP concentration in the absence of supernatant.

Estimation of exogenous adenosine. Supernatant samples from cultures of $B$. brevis were prepared by centrifuging and the adenosine content was determined using the following reaction mixture: phosphate buffer $\mathrm{pH} \mathrm{7.5,} 150 \mu \mathrm{mol}$; adenosine deaminase, $50 \mu \mathrm{g}$; supernatant sample, 0.2 to $0.5 \mathrm{ml}$; and water to $3.0 \mathrm{ml}$. The $E_{265}$ at $25^{\circ} \mathrm{C}$ was measured on a Unicam SP 800 recording spectrophotometer and adenosine determined by comparison with authentic standards.

Extraction and identification of acid-soluble nucleotides. Cultures were harvested at $13000 \mathrm{~g}$ and the supernatant retained for later analysis. $\mathrm{HClO}_{4}(20 \mathrm{ml}$ of $6 \% \mathrm{v} / \mathrm{v})$ was 


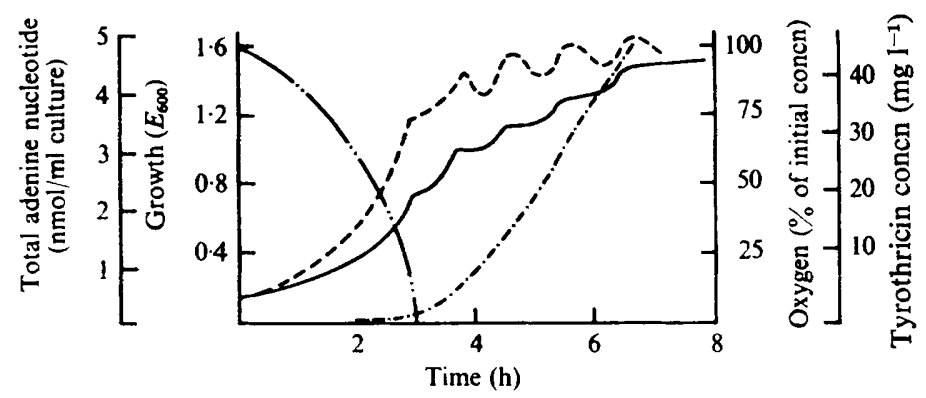

Fig. I. Changes in adenine nucleotide, oxygen and tyrothricin levels during growth of $B$. brevis under sealed culture conditions. (a) - Growth curve; an $E_{600}$ value of I.O represents $360 \mu \mathrm{g}$ bacterial dry $\mathrm{wt} / \mathrm{ml}$ culture. $(b)----$, Total adenine nucleotide; the values shown are the means of eight determinations. $(c)-. . .-$, Oxygen content of culture flask; the initial oxygen tension was that in equilibrium with air at $37^{\circ} \mathrm{C}$ and ambient pressure (approx. $760 \mathrm{mmHg}$ ). (d) $-\cdot-\cdots$, Tyrothricin production; the values shown are the means of 12 determinations.

added to the bacterial pellet and the mixture shaken for 10 min. Denatured protein was then removed by centrifuging at $15000 \mathrm{~g}$. The supernatant containing the acid-soluble nucleotides was immediately applied to a $\mathrm{I} 0 \times \mathrm{I} \mathrm{cm}$ diameter column packed with about $0.5 \mathrm{~g}$ activated charcoal (Norit). The culture supernatant, normally about $960 \mathrm{ml}$, was applied directly to a similar column.

Complete adsorption of the nucleotides on to the charcoal was checked by monitoring the $E_{260}$ values of the sample eluant and subsequent distilled water washings. Nucleotides were eluted from the charcoal with $15 \mathrm{ml}$ of $2 \mathrm{M}-\mathrm{NH}_{4} \mathrm{OH}$ in $6 \%(\mathrm{v} / \mathrm{v})$ ethanol. The eluant was then evaporated to dryness at $20^{\circ} \mathrm{C}$ by distillation. The residue was dissolved in $2 \mathrm{ml}$ of water, transferred to a specimen tube and dried under a stream of $\mathrm{N}_{2}$ at $40{ }^{\circ} \mathrm{C}$. It was then redissolved in $200 \mu \mathrm{l}$ of water and $10 \mu \mathrm{l}$ was chromatographed on Whatman no. I paper using the solvent system, isobutyric acid- $\mathrm{NH}_{4} \mathrm{OH}$-water $(66: \mathrm{I}: 33$, by vol.). Nucleotides were identified after u.v. visualization by comparison with authentic standards run under identical conditions. The concentrations were measured by cutting out the area of paper containing the nucleotide, eluting with $\mathrm{I} \mathrm{ml}$ of water acidified with $0 \cdot 1 \mathrm{ml}$ of $\mathrm{I} \mathrm{M}-\mathrm{HCl}$, and reading the $E_{280}$ on a Unicam SP 500 spectrophotometer. The concentration of an individual nucleotide was derived by comparing its $E_{260}$ value with the total extinction of the whole eluted chromatogram, which was for the present purpose taken to represent the entire nucleotide pool, and calculated from $E_{260}=15.4 \times 10^{3} 1 \mathrm{~mol}^{-1} \mathrm{~cm}^{-1}$ (Bergmeyer, 1962).

Addition of tyrothricin to growing cultures. Tyrothricin was injected aseptically into growing cultures as an ethanolic solution of $30 \mathrm{mg}$ tyrothricin $/ \mathrm{ml}$. The volume injected was adjusted as necessary with ethanol to establish a final concentration of $0.5 \%$ ethanol in the culture medium. A blank was also included where ethanol alone was injected to give a final concentration of $0.3 \%$ in the flask. The estimation of endogenous tyrothricin has been described previously (Seddon \& Fynn, I97I).

\section{RESULTS}

In a sealed culture flask, the organism utilized the available oxygen to grow typically to an $E_{600}$ of 0.75 , at which point the oxygen concentration reached zero (Fig. I $a, c$ ). Subsequent anaerobic growth was slower and exhibited periodic lag phases of increasing duration from Io $\mathrm{min}$ to $25 \mathrm{~min}$ to $40 \mathrm{~min}$ (Fig. I $a$ ), and during this time, tyrothricin production increased 


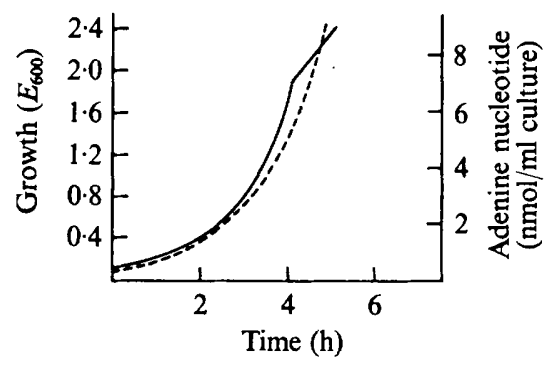

Fig. 2. Changes in adenine nucleotide level during growth of $B$. brevis under aerated culture conditions. , Growth curve; - - - , total adenine nucleotide.
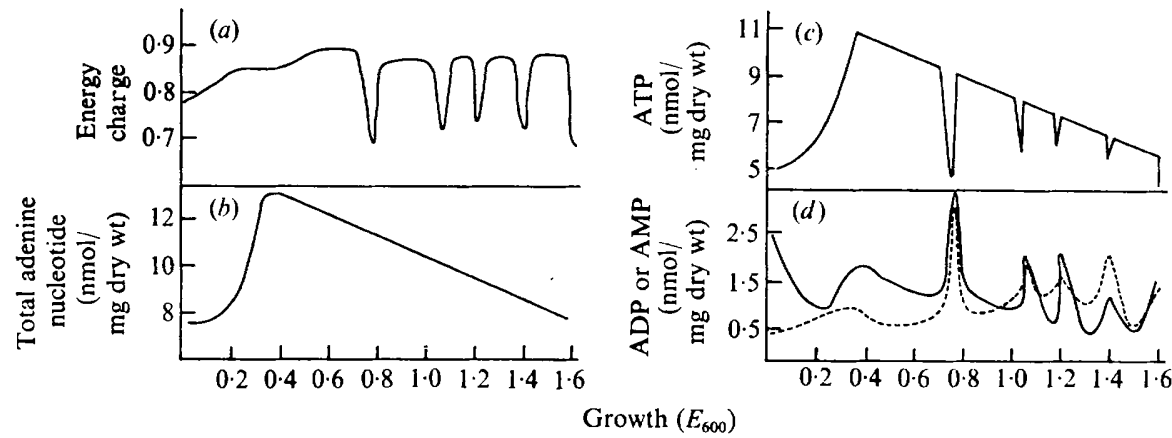

Fig. 3. Energy charge value $(a)$ and adenine nucleotide concentrations $(b, c, d)$ plotted as functions of growth of $B$. brevis under sealed culture conditions. In $(d)$, ADP and AMP concentrations are shown by the full and broken lines, respectively.

rapidly but without any detectable periodic variation in rate (Fig. I $d$ ). Growth ceased in the sealed system when the culture $E_{600}$ had reached I.56 (Fig. I $a$ ). In the continuously aerated culture the aerobic growth was rapid (Fig. 2) and tyrothricin production was suppressed. Growth under forced aeration continued beyond an $E_{600}$ of 2.5 , when the experiments were normally concluded.

The endogenous nucleotide levels during growth are shown in Figs. I (b), 2 and 3. In the aerated system the total adenine nucleotide concentration was constant at $9.5 \pm 1 \cdot 2$ $\mathrm{nmol} / \mathrm{mg}$ dry wt. The concentrations of the individual nucleotides, expressed in $\mathrm{nmol} / \mathrm{mg}$ dry wt, were AMP $0.8 \pm 0.4$, ADP I $0 \pm 0.5$, and ATP $7.7 \pm 0.9$; the E.C. value was stable at $0.87 \pm 0.02$. The concentration of adenine nucleotides during growth under sealed conditions was variable. One pattern of variation was linked to short periodic oscillations in anaerobic growth whose onset occurred at $E_{600}=0.75$. These lag periods were accompanied by a fall in the level of ATP and a rise in the level of ADP and AMP; thus the E.C. value fell to a value of 0.70 during lag phases compared with a value of 0.87 during growth phases (Figs. $\mathrm{I} a$ and $3 a$ ). There was also a longer term variation in total adenine nucleotide, linked to the growth cycle of the organism grown under sealed conditions. The concentration of total nucleotide increased from a value of $7.5 \mathrm{nmol} / \mathrm{mg}$ dry wt at the commencement of growth to a maximum value of $12.5 \mathrm{nmol} / \mathrm{mg}$ at an $E_{600}$ of 0.35 to 0.40 , and then declined to approximately the initial value when growth finally ceased (Figs. I $a$ and $3 b$ ). The manifest loss of total adenine nucleotide from an $E_{600}$ of 0.4 onwards was evident as a discontinuity in the curve when total nucleotide/ml culture was plotted against time (Fig. I $b$ ). The 
Table I. Comparison of acid-extractable nucleotides from B. brevis ATCCI0068 and B. brevis GB

B. brevis ATCCI0068

*Nucleotide $\begin{gathered}\text { Tyrothricin- } \\ \text { producing }\end{gathered} \begin{gathered}\text { Non-tyrothricin- } \\ \text { producing }\end{gathered}$

\section{AMP}

GMP

GMP-peptide

Nucleotide-peptide

$\begin{array}{lr}54 & 75 \\ \text { I4 } & 0 \\ \text { II } & 0 \\ \text { 2I } & 20\end{array}$

\begin{tabular}{lcc}
\multicolumn{3}{c}{ B. brevis GB $\dagger$} \\
Nucleotide & $\begin{array}{c}\text { Gramicidin- } \\
\text { producing }\end{array}$ & $\begin{array}{c}\text { Non-gramicidin- } \\
\text { producing }\end{array}$ \\
AMP & 6 & 0 \\
GMP & 40 & 55 \\
GMP-peptide & 20 & 0 \\
UMP-peptide & 10 & 10
\end{tabular}

* Nucleotide values expressed as percentages of the total nucleotide pool.

+ Data of Silaeva et al. (1965). For the purposes of comparison, the adenine nucleotide-containing compounds have been included with AMP.

organism grown under forced aeration showed no such variations, the total nucleotide/mg dry wt of bacteria remaining essentially constant during growth (Fig. 2).

Four nucleotide-containing compounds (I to 4 ) with $R_{F}$ values respectively of $0.31,0.47$, 0.49 and 0.56 were obtained by chromatography (see Methods) of the acid-soluble extract of $B$. brevis grown under conditions of tyrothricin production. The $R_{F}$ values of compounds (3) and (4) corresponded to those obtained with authentic AMP and GMP standards respectively. Compounds (1) and (2) were also ninhydrin positive and fluorescent at $340 \mathrm{~nm}$, suggesting that (I) and (2) were nucleotide-peptides with the possibility that one or other was a GMP-peptide (perhaps originally present in the growing culture as a GTP-peptide). This would explain the appearance of free GMP, split off the peptide as an artefact of the extraction procedure and subsequently degraded in the same way as adenine nucleotides are known to be degraded to the monophosphate in this organism (Davison \& Fynn, 1974). Comparison of the extracts prepared under tyrothricin-producing and non-producing conditions showed that GMP and one of the nucleotide-peptides (compound I) were not present in the absence of tyrothricin. This observation suggests that compound (I) is a GMP-peptide analogous to that found by Silaeva et al. (1965). Similar changes occurred in the GMP-peptide content of the nucleotide pool in both tyrothricin- and gramicidin Sproducing strains in the presence and absence of antibiotic production (Table I).

When $2.3 \mathrm{mg}$ tyrothricin $/ \mathrm{ml}$ were injected into growing $B$. brevis cultures there was an immediate inhibition of growth which lasted for 10 to $15 \mathrm{~min}$, increasing to several hours when $30 \mathrm{mg} / \mathrm{l}$ was used. Addition of $45 \mathrm{mg} / \mathrm{l}$ permanently inhibited further growth. Analysis of the medium by the luciferin-luciferase method immediately following tyrothricin injection revealed the presence of a large amount of adenine nucleotide; this is rapidly degraded to adenosine by extracellular alkaline phosphatase activity in the medium (Davison \& Fynn, 1974). A maximum of $2 \mathrm{nmol}$ adenine nucleotide/ $\mathrm{ml}$ of medium was detected following injection, using the most rapid sampling procedure practicable. The total adenine nucleotide detectable in the medium fell as the concentration of tyrothricin injected was reduced. Where very low amounts of tyrothricin ( $\mathrm{I}$ to $2 \mathrm{mg} / \mathrm{l}$ of culture) were injected, little change in total cellular adenine nucleotide was observed although significant losses could be detected when increased amounts of antibiotic were injected. Exogenous tyrothricin also caused E.C. values to fall gradually to between 0.64 and 0.70 during a 5 min period following injection. The E.C. value returned to normal when growth was resumed. Increasing the amount of tyrothricin injected increased both the duration of the induced lag phase and the loss of nucleotide from the organisms. When $45 \mathrm{mg}$ or more of tyrothricin/l was used there was an 
apparent total loss of adenine nucleotide from the bacteria and no further growth. The aerated and sealed cultures responded in an identical way to injected tyrothricin: equal amounts of antibiotic injected produced the same lag period in growth, and $45 \mathrm{mg}$ tyrothricin/l completely inhibited growth in both cultures.

\section{DISCUSSION}

The values at ATP, total nucleotide and E.C. for B. brevis growing aerobically are in good agreement with published values for other micro-organisms (Chapman et al., I971; Cole, Wimpenny \& Hughes, 1967; Chin \& Bernstein, 1968; Decker \& Pfitzer, 1972; Dietzler, Leckie \& Lais, I973; Knowles \& Smith, 1970; Miovic \& Gibson, I973; Harrison \& Maitra, 1969; Robertson \& Wolfe, 1970; Scherbaum et al., 1962; Slayman, 1973; Strange, Wade \& Dark, 1963). In the few reports where the levels of individual nucleotides have been investigated, these do not resemble the nucleotide pattern found in $B$. brevis growing under sealed culture conditions. The transition from aerobic to anaerobic metabolism in $B$. brevis is accompanied by a slower growth rate with periodic lag phases, a rapid increase in the concentration of endogenous tyrothricin and an apparent depletion of cellular nucleotide.

Gramicidin $\mathrm{S}$ is structurally a member of the tyrocidine group (Hunter \& Schwartz, 1967), and stimulates the release of cellular material from bacteria (Silaeva et al., 1965). The observed fall in endogenous nucleotide levels at the onset of anaerobic growth in $B$. brevis could be a consequence of the release of these compounds into the external medium.

An extracellular nucleotide-degrading enzyme secreted by $B$. brevis (Davison \& Fynn, 1974) rapidly converts adenine nucleotides to adenosine. This prevents direct determination of extracellular nucleotide although no adenosine or any other compound with significant extinction at $260 \mathrm{~nm}$ was detected in media from $B$. brevis culture.

Glazer, Silaeva \& Shestakov (1966), have suggested that the formation of nucleotidepeptides is associated with antibiotic production. Silaeva et al. (I965) have shown that a GMP-peptide contributing $20 \%$ of the total cell nucleotide is formed only during gramicidin $\mathrm{S}$ synthesis in $B$. brevis $\mathrm{GB}$, and a similar increase in GMP-peptide was observed during antibiotic production by the ATCCI0068 strain. The observed depletion of endogenous adenine nucleotide could therefore be a consequence of increased utilization in the formation of nucleotide-peptide.

The initial fall in E.C. value and subsequent lag phase corresponded to the depletion of oxygen in the sealed system and presumably reflected the transition to an anaerobic mode of energy production. The increase in antibiotic concentration was linear, uninterrupted by any cyclic variations (Fig. I $c$ ). Thus if tyrothricin were inhibiting energy production, presumably through the reported effects on membrane structure (Neubert \& Lehninger, 1962), then it seems unlikely that the observed recoveries in E.C. value would have occurred. The failure to find adenosine in the culture medium during tyrothricin production also suggests that membrane structure is not markedly impaired. The amount of endogenous tyrothricin present $(45 \mathrm{mg} / \mathrm{l})$ at the point of growth cessation in a sealed culture of $B$. brevis prevented growth when added exogenously to both sealed and aerated (tyrothricin-free) growing cultures. Since the sealed culture produced endogenous tyrothricin it might be expected that the amount of exogenous tyrothricin required to inhibit growth would be less than in the aerated system. Bacillus brevis cultures grown under limited aeration also produced more than $45 \mathrm{mg}$ tyrothricin/l. These observations suggest that endogenous tyrothricin is not the agent responsible for the cessation of growth of the organism under anaerobic conditions and implies that the antibiotic does not inhibit the energy-producing mechanisms to any 
marked extent and hence is not responsible for the oscillation observed in E.C. and growth. It seems more plausible that the variation of E.C. and growth rate on initiation of anaerobic growth in $B$. brevis are manifestations of regulatory processes involving, for example, glycolytic enzymes, as suggested by Atkinson (1968). Preliminary investigations have shown the presence of glycolytic enzymes capable of utilizing glycerol to form pyruvate (E. E. Anato-Dumelo \& G. H. Fynn, unpublished), although we have not yet established if anaerobic glycolysis is a functional metabolic pathway in this organism.

\section{REFERENCES}

Atrinson, D. E. (1968). Citrate and the citrate cycle in the regulation of energy metabolism. In The Metabolic Roles of Citrate, pp. 23-40. Edited by T. W. Goodwin. London and New York: Academic Press.

BergmeYer, H. H. (1962). Methods of Enzymatic Analysis, p. 1005. London and New York: Academic Press.

Chapman, A. G., Fall, L. \& Atkinson, D. E. (1971). Adenylate energy charge in Escherichia coli during growth and starvation. Journal of Bacteriology 108, $1072-1086$.

ChIN, B. \& BerNSTEIN, I. A. (1968). ATP and synchronous mitosis in Physarum polycephalum. Journal of Bacteriology 96, 330-337.

Cole, H. A., Wimpenny, J. W. T. \& Hughes, D. E. (1967). The ATP pool in E. coli. I. Measurement using a modified luciferase assay. Biochimica et biophysica acta 143, 445-453.

Davison, J. A. \& FYNN, G. H. (1974). Interference by phosphates in ATP estimation by the luciferase method. Analytical Biochemistry 58, 632-637.

DeCKER, K. \& Pfizer, S. (1972). Determination of steady state concentrations of adenine nucleotides in growing Clostridium kluyveri cells by biosynthetic labelling. Analytical Biochemistry 50, 529-539.

DietzleR, D. N., LeCKIE, M. P. \& LAIS, C. J. (I973). Rates of glycogen synthesis and the cellular levels of the ATP and FDP during exponential growth and the nitrogen limited stationary phase of $E$. coli W4597(K). Archives of Biochemistry and Biophysics 156, 684-693.

FYNN, G. H. \& SEDdON, B. (1971). Terminal oxidations in Bacillus brevis. III. Evidence of TCA cycle enzyme activity. Archiv für Mikrobiologie 79, 274-279.

FynN, G. H., ThomAs, D. V. \& SedDon, B. (1972). On the role of menaquinone in the reduced nicotinamide adenine dinucleotide oxidative pathway of Bacillus brevis. Journal of General Microbiology 7o, 27I-275.

Glazer, V. M., Silaeva, S. A. \& Shestakov, S. V. (1966). The effect of gramicidin S on phosphorus metabolism in Bacillus brevis. Biokhimiya 3I, II 35-1141.

HarRison, D. E. F. \& MaITRA, P. K. (1969). Control of respiration and metabolism in growing Klebsiella aerogenes. The role of adenine nucleotides. Biochemical Journal 112, 647-656.

Hunter, F. E. \& SchwarTz, L. S. (I967). In Antibiotics, vol. I, pp. 636-64I. Edited by D. Gottlieb and P. D. Shaw. Berlin: Springer-Verlag.

KNOWLES, C. J. \& SMITH, L. (1970). Measurement of ATP levels of intact Azotobacter vinelandii under different conditions. Biochimica et biophysica acta 197, I 52-160.

Miovic, M. L. \& Gibson, J. (1973). Nucleotide pools and adenylate energy charge in balanced and unbalanced growth of Chromatium. Journal of Bacteriology 114, 86-95.

Neubert, D. \& Lehninger, A. L. (1962). The effect of oligomycin, gramicidin and other antibiotics on reversal of mitochondrial swellings by adenosine triphosphate. Biochimica et biophysica acta 62, 556-565.

Robertson, A. M. \& Wolfe, R. S. (1970). ATP pools in Methanobacterium. Journal of Bacteriology roz, 43-51.

Scherbaum, O. H., Chou, C. L., Saraydarian, K. H. \& Byfield, J. E. (I962). The effect of temperature shifts on the intracellular level of nucleoside triphosphates in Tetrahymena pyriformis. Canadian Journal of Microbiology 8, 753-760.

SedDon, B. \& FynN, G. H. (1970). Terminal oxidations in Bacillus brevis atccioo68. I. Measurement of the NADH oxidase activity. Biochimica et biophysica acta 216, 435-438.

SEDDON, B. \& FYNN, G. H. (1971). Terminal oxidations in Bacillus brevis. II. The electron transport systems. Archiv für Mikrobiologie 77, 252-26I.

SEDDON, B. \& FYNN, G. H. (1973). Energetics of growth in a tyrothricin-producing strain of Bacillus brevis. Journal of General Microbiology 74, 305-314.

Silaeva, S. A., Glazer, V. M., Shestakov, S. V. \& Prokofiev, M. A. (1965). Nucleotides of Bacillus brevis G.B. cells producing and not producing gramicidin S. Biokhimiya 30, 947-955.

Slayman, C. L. (1973). Adenine nucleotide levels in Neurospora as influenced by conditions of growth and by metabolic inhibitors. Journal of Bacteriology 114, 752-766.

Strange, R. E., WADE, H. R. \& Dark, F. A. (I963). The effect of starvation on the ATP concentration in Aerobacter aerogenes. Nature, London 199, 55-57. 